\title{
An Intelligent Method for Evaluation of Production Scheduling Performance
}

\author{
Wei $\mathrm{Xu}^{1 \mathrm{1a}}$, Jiarong Yang, Raofen Wang ${ }^{2 *}$ \\ ${ }^{1}$ Central Academe, Shanghai Electric Group Co., Ltd. Shanghai, China \\ ${ }^{2}$ College of Electronic and Electrical Engineering, Shanghai University of Engineering \\ ScienceShanghai, China \\ ae-mail: xuweisec@126.com, *email: rfwangsues@163.com
}

\begin{abstract}
Keywords: league championship algorithm; match schedule; production scheduling
\end{abstract}
\begin{abstract}
To predict the production scheduling performance in a shop floor, an intelligent method based on a modified league championship algorithm is proposed. The method overcomes the problems like premature, being stuck to local minima, etc. A new match schedule is introduced to enhance the competition capability for every single team. One scheme for updating the team member is presented to diversify the population. The application of the proposed method on the production scheduling showed that the novel approach could provide accurate prediction of scheduling performance indexes and is capable of being used in the real world shop floor.
\end{abstract}

\section{Introduction}

Since genetic algorithm [1] inspired by the process of natural selection was proposed by Professor Holland in 1975, artificial intelligent computing has made much progress. Kirkpatrick et al showed how the simulated annealing method [2] for approximating numerical simulation of the behavior of a many-body system at a finite temperature and applied it on combinatorial optimization. Tabu search [3, 4], created by Fred W. Glover formalized in 1989 is a metaheuristic search method employing local search methods used for mathematical optimization. Dr. Dorigo [5] presented ant colony optimization inspired by the real ant colonies foraging. In 1995, originated from the flocking behavior of birds, particle swarm optimization (PSO) [6] was proposed for complex optimization problems. In decades, the intelligent optimization algorithms have widely been applied in many areas, such as transportation [7], power [8], Communication [9], Manufacturing [10], and so on.

League championship algorithm (LCA) proposed by Dr. Kashan [11] is a novel population based algorithmic framework for global optimization. The core idea of LCA is originated from the competition process of league teams. However, the original LCA is still easily being stuck to local optima, which causes that the global exploration and local exploitation are not balanced. In this paper a modified LCA method is proposed to avoid the above issues, and is applied on the scheduling performance prediction in a practical plant.

\section{Original League Championship Algorithm}

LCA has a population called "league" with L sport teams. Each team is composed of D individuals. The team who has the best "playing strength" $\mathrm{f}$ is the champion. The formation of team i $\left({ }^{i \in\{1,2, \ldots, L\}}\right)$ in iteration $\mathrm{t}\left({ }^{t \in\{1,2, \ldots,(L-1) \times S\}}\right)$ is $X_{i}^{t}=\left\{x_{i 1}^{t}, x_{i 2}^{t}, \ldots, x_{i d}^{t}, \ldots ., x_{i D}^{t}\right\}$ where $\mathrm{S}$ is the season amount, and the best formation of team $\mathrm{i}$ until iteration $\mathrm{t}$ is $B_{i}^{t}=\left\{b_{i 1}^{t}, b_{i 2}^{t}, \ldots, b_{i d}^{t}, \ldots ., b_{i D}^{t}\right\}$ 。

The match schedule must be preset first. A single round-robin schedule is utilized in which each team competes against every other team once in each season. In order to win the every match, each team has to design the formation according to the competitor which is played against. The SWOT matrix is employed. The strengths (S) and weaknesses (W) of the team as well as the opportunities (O) and threats (T) are focused.

Assume that team $\mathrm{i}$ has competed against team $\mathrm{j}$, and team $\mathrm{l}$ against team $\mathrm{k}$ at round $\mathrm{t}$. The 
updating way at round $t+1$ is given by:

$$
x_{i d}^{t+1}=\left\{\begin{array}{r}
b_{i d}^{t}+y_{i d}^{t}\left(c_{1} r_{1}\left(x_{i d}^{t}-x_{k d}^{t}\right)+c_{1} r_{2}\left(x_{i d}^{t}-x_{j d}^{t}\right)\right) \\
\quad \text { if } f\left(x_{i}^{t}\right)>f\left(x_{j}^{t}\right) \cap f\left(x_{l}^{t}\right)>f\left(x_{k}^{t}\right) \\
b_{i d}^{t}+y_{i d}^{t}\left(c_{2} r_{1}\left(x_{k d}^{t}-x_{i d}^{t}\right)+c_{1} r_{2}\left(x_{i d}^{t}-x_{j d}^{t}\right)\right) \\
\quad \text { if } f\left(x_{i}^{t}\right)>f\left(x_{j}^{t}\right) \cap f\left(x_{k}^{t}\right)>f\left(x_{l}^{t}\right) \\
b_{i d}^{t}+y_{i d}^{t}\left(c_{1} r_{2}\left(x_{i d}^{t}-x_{k d}^{t}\right)+c_{2} r_{1}\left(x_{j d}^{t}-x_{i d}^{t}\right)\right) \\
\quad \text { if } f\left(x_{j}^{t}\right)>f\left(x_{i}^{t}\right) \cap f\left(x_{l}^{t}\right)>f\left(x_{k}^{t}\right) \\
b_{i d}^{t}+y_{i d}^{t}\left(c_{2} r_{2}\left(x_{k d}^{t}-x_{i d}^{t}\right)+c_{2} r_{1}\left(x_{j d}^{t}-x_{i d}^{t}\right)\right) \\
\quad \text { if } f\left(x_{j}^{t}\right)>f\left(x_{i}^{t}\right) \cap f\left(x_{k}^{t}\right)>f\left(x_{l}^{t}\right)
\end{array}\right.
$$

where $\mathrm{c} 1$, c2 represent the preset weights in the range of $[0,1] ; \mathrm{r} 1, \mathrm{r} 2$ are random numbers distributed uniformly in [0,1]; $y_{i d}^{t}$ is binary number which determines if dth dimension of $X_{i}^{t}$ is updated. The updating amplitude of team i $q_{i}^{t}$ is

$q_{i}^{t}=\sum_{d=1}^{D} y_{i d}^{t}$

$q_{i}^{t}$ is calculated by using the truncation geometric distribution method[12], which is defined as follows:

$$
q_{i}^{t}=\left\lceil\frac{\ln \left(1-\left(1-\left(1-p_{c}\right)^{n}\right) r\right)}{\ln \left(1-p_{c}\right)}\right\rceil
$$

where $\mathrm{r}$ is random number in the range of $[0,1]$, pc is the preset parameter in $(0,1)$.

\section{Modifications on League Championship Algorithm}

In the modified LCA (MLCA), the competitor is different for each team at each season. The new schedule rules are shown as Figure 1, in which $\mathrm{n}$ is the team number fixed in current season, and the schedule arrangements of other teams change in clockwise.

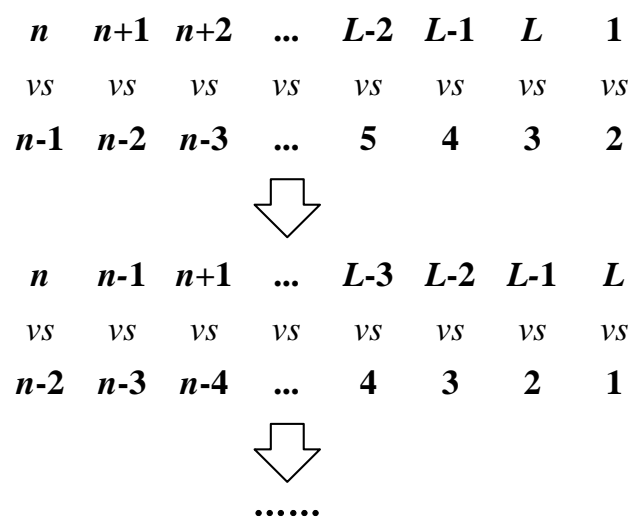

Figure 1. $\quad$ New match schedule

Additionally, a free search (FS) operation is introduced into the league competition in iteration $\mathrm{k}$ randomly. The $x_{i}^{k}$ is chosen as the start location $z_{i}^{0}$ in the FS operation. Then the exploration walk generates coordinates of a new location $z_{i}^{m}$ as

The modification strategy is

$$
z_{i j}^{m}=z_{i j}^{0}-2 \Delta z_{i j}^{m} \times \operatorname{random}_{i j}^{t}(0,1)
$$

$$
\Delta Z_{i j}^{m}=R_{i j} \times\left(X_{\max j}-X_{\min j}\right) \times \operatorname{random}_{i j}^{t}(0,1)
$$

where Rij is the individual neighbor space; $\mathrm{m}$ is the current step, $\mathrm{m}=1,2, \ldots, \mathrm{T}$; $\mathrm{T}$ is the step limit per walk, which is preset. 


\section{Prediction on Production Scheduling Performance}

In a shop floor of a discrete plant, the production scheduling problem is studied in order to further prove the effectiveness of the modified LCA method. The product from the shop floor is composed of ten parts and four subassemblies which would be machined and assembled through many steps. Under given conditions, such as material availability, machine availability, machine downtime, unit processing time, etc., the scheduler's decisions influence the key performance index of production scheduling, i.e. the working time completion rate for 14 parts or subassemblies.

A mathematical model with feed-forward neural network (NN) structure is established to describe the complex nonlinear relationship between scheduler's decisions and the working time completion rate. The parameters of the model is identified by using the proposed MLCA. The parameter settings of MLCA-NN are in the following: league size $L=30$; season amount $S=100$; $\mathrm{c} 1=\mathrm{c} 2=0.5 ; \mathrm{pc}=0.01$. The training and testing results based on MLCA-NN are illustrated in Figure 2. The figures indicate that MLCA-NN could fit the actual values well and is capable of predicting the working time completion rate. The good generalization is satisfying.
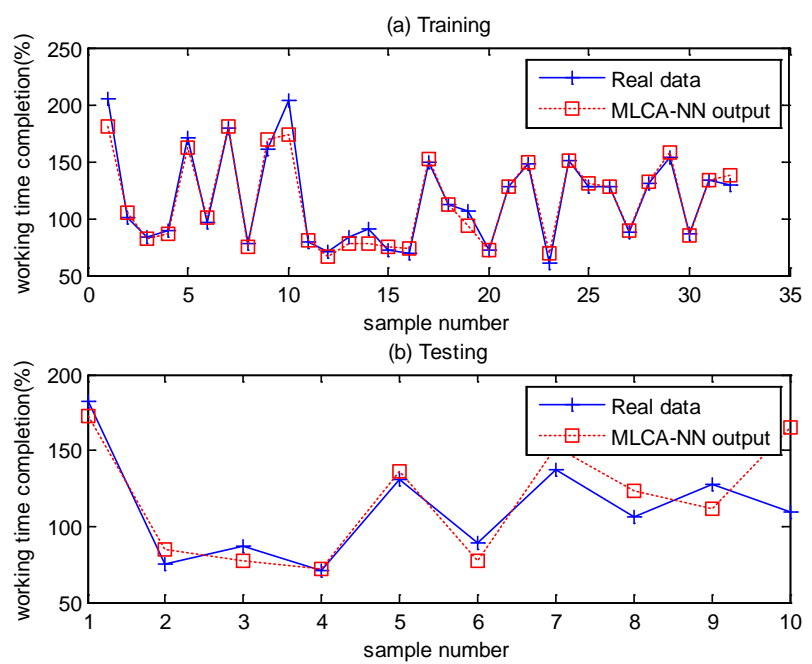

Figure 2. $\quad$ Results of MLCA-NN for working time completion rate

For further evaluating the performance of MLCA, PSO and LCA combined with the same structure of neural network as above are employed to establish two single scheduling models. The mean square error (MSE) comparison is illustrated in Table I. In general, the generalization capability is the primary concern. It is illustrated that the MSE of MLCA-NN for test purpose is much smaller than those of PSO-NN and LCA-NN though its training error is larger. For the working time completion rate, MSE of the testing results by using MLCA-NN is reduced by $56.16 \%, 41.34 \%$ in the comparison with PSO-NN and LCA-NN respectively. The comparison results imply that the MLCA-NN model could provide reliable prediction on production scheduling performance and is suitable for application in the real-world shop floor.

TABLE I. MEAN SQUARE ERROR COMPARISON OF WORKING TIME COMPLETION RATE

\begin{tabular}{|c|l|l|l|l|l|}
\hline \multicolumn{2}{|c|}{ PSO-NN } & \multicolumn{2}{c|}{ LCA-NN } & \multicolumn{2}{c|}{ MLCA-NN } \\
\hline Training & Testing & Training & Testing & Training & Testing \\
\hline 7.9285 & 28.0454 & 8.5701 & 20.9607 & 8.4197 & 12.2951 \\
\hline
\end{tabular}

In general, the neuron amount in the hidden layer is very critical for the prediction and generalization capability of the neural network. Thus, a separate simulation on choosing different neuron amount of hidden layer is carried out. The performance of MLCA-NN with different amount of hidden neuron is shown in Table II. Along with the increasing of hidden neuron, the computing time by using the proposed model grows dramatically as shown in Figure 3. Meantime, the MSE for training data reduces while the testing MSE increases overall, illustrated in Figure 4. The analysis 
implies that different hidden neuron amount affects the performance of neural network greatly. It is necessary for good generalization capability of $\mathrm{NN}$ to choose appropriate neuron amount in hidden layer.

TABLE II. PERFORMANCE OF MLCA-NN WITH DIFFERENT AMOUNT OF HIDDEN NEURON

\begin{tabular}{|c|c|c|c|}
\hline Neuron amount & Computing time (s) & MSE for training (\%) & MSE for testing (\%) \\
\hline 5 & 101 & 9.4655 & 8.1295 \\
\hline 10 & 190 & 8.4197 & 12.2951 \\
\hline 15 & 270 & 7.6865 & 25.9107 \\
\hline 20 & 337 & 7.5528 & 16.3594 \\
\hline 25 & 430 & 6.3409 & 27.5224 \\
\hline 30 & 511 & 6.2843 & 9.2587 \\
\hline 40 & 669 & 5.0224 & 29.9955 \\
\hline 50 & 845 & 4.9429 & 21.2959 \\
\hline 60 & 966 & 4.9080 & 27.8863 \\
\hline 80 & 1349 & 5.0923 & 26.6281 \\
\hline 100 & 1665 & 5.4170 & 37.0445 \\
\hline 150 & 2544 & 4.9145 & 27.8586 \\
\hline 200 & 3619 & 4.8092 & 39.0638 \\
\hline
\end{tabular}
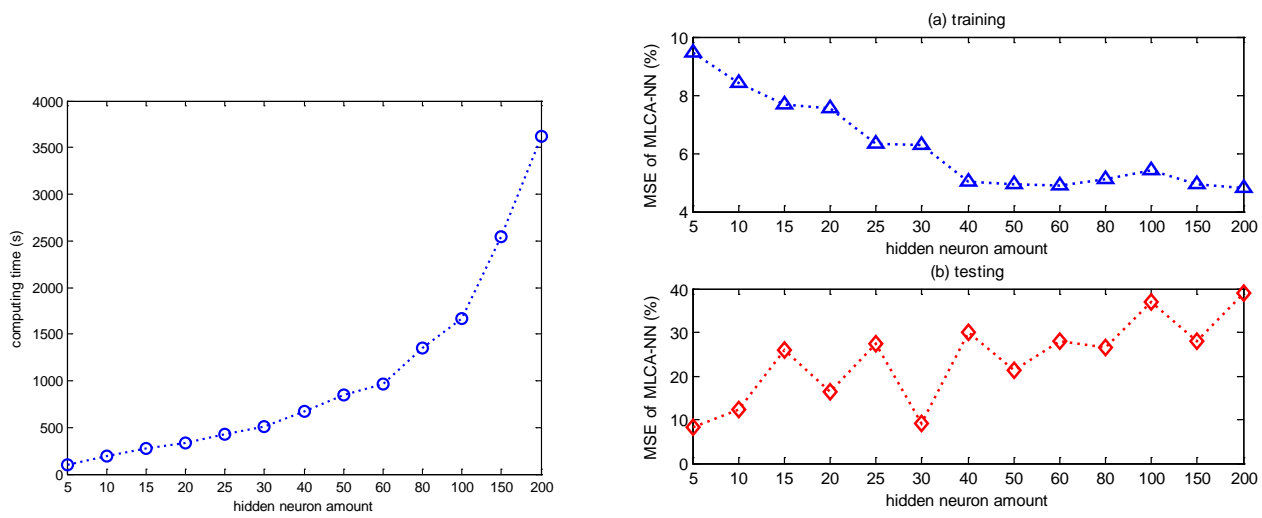

Figure 3. Computing time by using MLCA-NN with different hidden neuron amount

Figure 4. $\quad$ MSE by using MLCA-NN with different hidden neuron amount for (a) traning data and (b) testing data

In addition, for MLCA method, the league size and season amount are two important adjustable parameters. Actually, the competition round number that is the product of league size and season amount reflects the behavior of latter two parameters. The MLCA-NN with different round number is studies for test purpose and the performance is shown in Table III. Similar to the variety of hidden neuron, the computing time increases along with the growth of round number in Figure 5. The training MSE reduces gradually, but the testing MSE is nearly unchanged when the round number exceeds 1500 as shown in Figure 6. Therefore, the small round number should be chosen to save the time consumption. 
TABLE III. PERFORMANCE OF MLCA-NN WITH DIFFERENT ROUND NUMBER (LEAGUE SIZE×SEASON AMOUNT)

\begin{tabular}{|c|c|c|c|}
\hline Round number & Computing time (s) & MSE for training (\%) & MSE for testing (\%) \\
\hline 500 & 4 & 17.2158 & 21.7993 \\
\hline 1000 & 19 & 17.4251 & 16.0437 \\
\hline 1500 & 44 & 13.9136 & 19.1300 \\
\hline 2000 & 81 & 11.0545 & 13.7354 \\
\hline 2500 & 126 & 9.2397 & 14.4973 \\
\hline 3000 & 190 & 8.4197 & 12.2951 \\
\hline 4000 & 324 & 7.1891 & 10.6311 \\
\hline 5000 & 496 & 5.9056 & 17.0042 \\
\hline 8000 & 1344 & 5.8492 & 11.0587 \\
\hline 10000 & 2039 & 5.8314 & 12.1085 \\
\hline
\end{tabular}
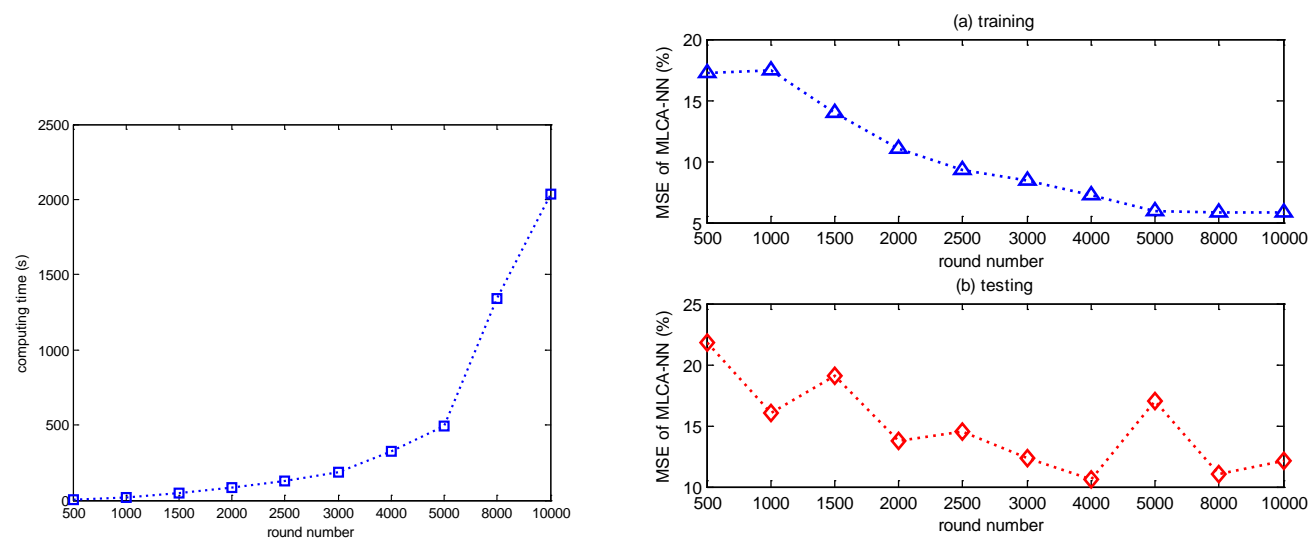

Figure 5. $\quad$ Computing time by using MLCA-NN with different round number

Figure 6. MSE by using MLCA-NN with different round number for (a) traning data and (b) testing data

\section{Conclusion}

A modified league championship algorithm is proposed to solve the drawbacks of the original algorithm like premature, slow convergence speed, etc. A novel match schedule is designed to diversify the formation of the sports teams. Free search operation is introduced to balance the global exploration capability and local exploitation capability. The MLCA is further used to evaluate the production scheduling performance in a practical shop floor. The results verified that the MLCA based neural network is very suitable for prediction on production plan and scheduling performance.

\section{Acknowledgment}

The work was supported by key science and technology project of Shanghai Municipal Science and Technology Commission (13DZ1101600).

\section{References}

[1] J. H. Holland, Adaptation in Natural and Artificial System. Michigan University Press, 1975.

[2] S. Kirkpatrick, C. D. Gelatt, and J. M. P. Vecchi, “Optimization by Simulated Annealing,” Science, vol. 220, May. 1983, pp. 671-680. 
[3] F. Glover, “Tabu Search: Part 1,” ORSA Jouranl on Computing, vol. 1, 1989, pp. 190-206.

[4] F. Glover, “Tabu Search: Part 2,” ORSA Jouranl on Computing, vol. 2, 1989, pp. 4-32.

[5] M. Dorigo and C. Blum, “Ant Colony Optimization Theory: A Survey,” Theoretical Computer Science, vol. 344, 2005, pp. 243-278.

[6] J. Kennedy and R. C. Eberhart, "Particle Swarm Optimization,” Proc. IEEE International Conference on Neural Networks, IEEE Press, vol. 4, 1995, pp. 1942-1948.

[7] J. C. Goodson, “A Priori Policy Evaluation and Cyclic-order-based Simulated Annealing for the Multi-compartment Vehicle Routing Problem with Stochastic Demands,” European Journal of Operational Research, vol. 241, 2015, pp. 361-369.

[8] N. J. Hill and G. T. Parks, "Pressurized Water Reactor in-core Nuclear Fuel Management by Tabu Search,” Annals of Nuclear Energy, vol. 75, 2015, pp. 64-71.

[9] Z. Ye and H. Mohamadian, "Adaptive Clustering based Dynamic Routing of Wireless Sensor Networks via Generalized Ant Colony,” IERI Procedia, vol. 10, 2014, pp. 2-10.

[10] T. Navalertporn and N. V. Afzulpurkar, "Optimization of Tile Manufacturing Process using Particle Swarm Optimization,” Swarm and Evolutionary Computation, vol. 1, 2011, pp. 97-109.

[11] A. H. Kashan, "League Championship Algorithm: A New Algorithm for Numerical Function Optimization,” 2009 IEEE International Conference of Soft Computing and Pattern Recognition, IEEE Press, Dec. 2009, pp. 43-48.

[12] A. H. Kashan, B. Karimi, and F. Jolai, "Effective Hybrid Genetic Algorithm for Minimizing Makespan on a Single-batch Processing Machine with Non-identical Job Sizes,” International Journal of Production Research, vol. 44, 2006, pp. 2337-2360. 